\title{
Optimum growth window for InAs/GalnSb superlattice materials tailored for very long wavelength infrared detection
}

\author{
Heather J. Haugan, ${ }^{\text {a) }}$ Gail J. Brown, Krishnamurthy Mahalingam, and Larry Grazulis \\ Air Force Research Laboratory, Materials and Manufacturing Directorate, Wright-Patterson Air Force Base, \\ Ohio 45433 \\ Gary T. Noe, Nathan E. Ogden, and Junichiro Kono \\ Department of Electrical and Computer Engineering, Rice University, Houston, Texas 77005
}

(Received 19 November 2013; accepted 24 January 2014; published 7 February 2014)

\begin{abstract}
The authors report growth studies to develop an InAs/GaInSb superlattice (SL) material for very long wavelength infrared detection. They select a SL structure of $47.0 \AA \mathrm{InAs} / 21.5 \AA \mathrm{Ga}_{0.75} \mathrm{In}_{0.25} \mathrm{Sb}$ that is designed for the greatest possible detectivity, and tune growth conditions to achieve the best quality ternary material. Since the material quality of grown layers is particularly sensitive to extrinsic defects such as nonradiative recombination centers generated during the growth process, the authors investigate the effect of the growth temperature $\left(T_{\mathrm{g}}\right)$ on the spectral photoresponse (PR) and carrier recombination lifetime using photoconductivity and time-resolved differential reflectivity measurements. Results indicate that a molecular beam epitaxy growth process the authors developed produces a consistent energy gap around $50 \mathrm{meV}$, determined from the PR spectra, but the intensity of the spectra is sensitive to $T_{\mathrm{g}}$. For SLs grown at $T_{\mathrm{g}}$ between 390 and $470{ }^{\circ} \mathrm{C}$, the PR signal intensity gradually increases as $T_{\mathrm{g}}$ increases from 400 to $440^{\circ} \mathrm{C}$, reaching a maximum at $440^{\circ} \mathrm{C}$. Outside this growth window, the SL quality deteriorates very rapidly. However, the carrier recombination lifetime measured at $300 \mathrm{~K}$ was not sensitive to $T_{\mathrm{g}}$. Although the SL sample grown at $430^{\circ} \mathrm{C}$ produced the longest lifetime of $84 \mathrm{~ns}$, the average $300 \mathrm{~K}$ lifetime value remained around 74 ns. (C) 2014 American Vacuum Society.
\end{abstract}

[http://dx.doi.org/10.1116/1.4864746]

\section{INTRODUCTION}

The development of infrared (IR) detectors that can operate at a high temperature with a high sensitivity is important for IR imaging systems. ${ }^{1-3}$ In the very long wavelength IR (VLWIR) band, the InAs/GaInSb superlattice (SL) system is an excellent candidate for IR photodiodes with cutoff wavelengths beyond $15 \mu \mathrm{m}$, especially for operating temperatures above $15 \mathrm{~K} .{ }^{4,5}$ With increasing indium composition, the lattice constant of the GaInSb layer increases, and the resulting biaxial tension in the InAs layer lowers the conduction band, while the biaxial compression in the GaInSb layer raises the valence band. In addition, the band gap of the GaInSb layer decreases with increasing indium, which also reduces the separation of the electron and heavy-hole (HH) minibands. As a result, a very narrow band gap can be achieved with a smaller period ${ }^{6}$ for the InAs/GaInSb SL system, leading to a larger absorption coefficient due to enhanced electron and hole wavefunction overlap. More importantly, the strain creates a large splitting between the $\mathrm{HH}$ and light-hole (LH) bands in the SLs, which minimizes the hole-hole Auger recombination process and increases the minority carrier lifetime. Therefore, the Auger-limited minority carrier lifetime, detectivity, and operating temperature can be significantly improved. For example, Grein et al. ${ }^{7}$ demonstrated how a small change in the strain-induced HH-LH splitting can create huge differences in the calculated detectivities. Grein et al. designed two SLs with the same $80 \mathrm{meV}$ band

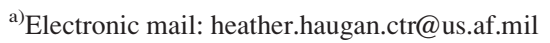

gap at $40 \mathrm{~K}$, but with different indium contents: $49.7 \AA$ InAs/57.0 $\AA \mathrm{Ga}_{0.9} \mathrm{In}_{0.1} \mathrm{Sb}$ and $47.0 \AA \mathrm{InAs} / 21.5 \AA \mathrm{Ga}_{0.75}$ $\mathrm{In}_{0.25} \mathrm{Sb}$. Based on the calculated electronic band structures for these two SL designs, the predicted total carrier lifetimes were $5 \times 10^{-9}$ and $1.4 \times 10^{-7} \mathrm{~s}$, respectively. This lifetime difference led to ideal device detectivities of $5.2 \times 10^{13}$ and $6.0 \times 10^{14}$ Jones, respectively, which demonstrates that an order of magnitude improvement in performance can be achieved by increasing the indium content. Therefore, from a theoretical perspective, the InAs/GaInSb system is more promising for VLWIR detection than the InAs/GaSb system.

Despite these theoretically predicted advantages, molecular beam epitaxy (MBE) growth parameters for the ternary SL materials are not as well established as for the binary SL materials. The majority of previously reported VLWIR photodiodes covering $50 \%$ cutoff wavelengths from 15 to $26 \mu \mathrm{m}$ were based on binary SL materials. ${ }^{8-11}$ The use of alloyed layers can lead to increased alloy scattering and can create a higher degree of disorder during epitaxial deposition such as indium segregation and compositional intermixing. Therefore, controlling the growth process to achieve a high-quality material with a precise band gap is more challenging for the ternary case. Although there was a report on a ternary SL photodiode with a 50\% cutoff wavelength of $21 \mu \mathrm{m}$ at $40 \mathrm{~K}$ using a 29 monolayers (ML) InAs/10 ML Ga $0_{0.93} \mathrm{In}_{0.07} \mathrm{Sb} \mathrm{SL}$ with $1 \mathrm{ML}$ of InSb-like interfacial bonds, ${ }^{12}$ the indium composition was too small to take full advantage of the potential strain benefits. The reported detectivity was only $3 \times 10^{9}$ Jones at $40 \mathrm{~K}$ for this photodiode, so the opportunity for significant improvements remains. 
In this work, using a combination of high-resolution x-ray diffraction (HRXRD), high-resolution transmission electron microscopy (HRTEM), atomic force microscopy (AFM), photoconductivity, and time-resolved differential reflectivity measurements, we optimized the MBE process for growing ternary InAs/GaInSb materials, specifically for VLWIR applications. We selected the SL design of $47.0 \AA$ InAs/ $21.5 \AA \mathrm{Ga}_{0.75} \mathrm{In}_{0.25} \mathrm{Sb}$ proposed by Grein et al., ${ }^{7}$ where their layer thicknesses and indium composition were adjusted to maximize the detectivity. Since the quality of SLs strongly depends on the densities of nonradiative recombination centers ${ }^{13-16}$ growth parameters such as the V/III flux ratio, growth rates of Group III elements, Group V cracking temperature, and interface shutter sequence were carefully coordinated to minimize the defect density and then tune the growth temperature within a preset growth condition to produce the highest-quality ternary SL materials.

\section{SUPERLATTICE GROWTHS}

The ternary SL materials were grown by a Varian GEN-II MBE machine equipped with dual-filament SUMO cells for the Group III elemental solid sources of Ga and In, and EPI valved cracker cells for $\mathrm{As}_{2}$ and $\mathrm{Sb}_{2}$. The repeated SL stacks $(0.5 \mu \mathrm{m}$-thick) and the undoped GaSb buffer layer ( $0.5 \mu \mathrm{m}$-thick) were deposited on lightly-doped n-type $\mathrm{GaSb}$ (100) wafers, and a series of $47.0 \AA$ In $A s / 21.5 \AA \mathrm{Ga}_{0.75} \mathrm{In}_{0.25}$ $\mathrm{Sb}$ SL samples were grown at a variety of growth temperatures $\left(T_{\mathrm{g}}\right)$, ranging from 390 to $470^{\circ} \mathrm{C}$. To grow the intended sample structure under minimum cross contamination environment of the anion fluxes, the V/III flux ratio was set at a minimum of 3 for both GaInSb and InAs layer depositions. In order to avoid an excess amount of As-for-Sb exchange that degrades layer qualities, ${ }^{17,18}$ we used a very slow growth rate for InAs layer deposition to minimize the As-background flux during the SL growth. Growth rates of 0.3 and $1.6 \AA / s$ were used for InAs and GaInSb layers, respectively. Since monomeric $\mathrm{Sb}$ is the species critical to reducing densities of nonradiative recombination centers in the SL material, ${ }^{19}$ we set the $\mathrm{Sb}$ cracking zone temperature at $950^{\circ} \mathrm{C}$ for the series, which is close to the suggested value by the EPI Model $200 \mathrm{cc}$ Mark V Corrosive Series Valved Cracker, and the As cracking zone temperature at $900{ }^{\circ} \mathrm{C} .{ }^{20}$ The interface type between layers was not intentionally controlled in order to compensate the residual strain in the ternary SLs. However, the net residual strain of our series was small and varied between $+0.2 \%$ and $0.0 \%$.

\section{RESULTS AND DISCUSSION}

\section{A. Structural quality}

In order to evaluate structural properties of grown samples, structural parameters such as the SL period, SL net strain, strain profiles across interfaces, and individual layer thickness were retrieved from a combination of HRXRD and HRTEM analyses. Figure 1 shows a typical diffraction pattern of a 47.0 $\mathrm{InAs} / 21.5 \AA \mathrm{Ga}_{0.75} \mathrm{In}_{0.25} \mathrm{Sb}$ SL structure in the series, showing well-defined satellite peaks and Pendellösung fringes around the satellite peaks, which demonstrate structural high

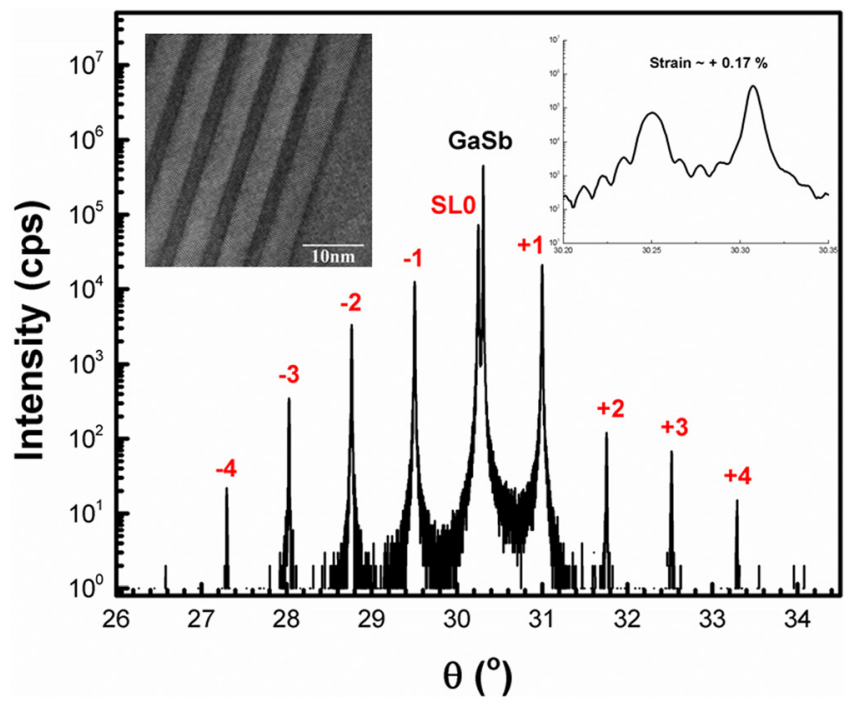

FIG. 1. (Color online) X-ray diffraction patterns of a compressively strained $68.0 \AA$ period ternary SL sample containing a $0.5 \mu \mathrm{m}$ thick $47.0 \AA$ InAs/21.5 $\mathrm{Ga}_{0.75} \mathrm{In}_{0.25} \mathrm{Sb}$ SLs. Inset is a HRTEM image of this including the first few layers near the substrate.

quality. To determine the SL period, the spacing between the SL satellite peaks was used. The period and strain were measured to be $68.0 \pm 0.5 \AA$ and $\sim+0.2 \%$, respectively. While the SL periods can be accurately determined by HRXRD, it is difficult to accurately measure the individual layer thicknesses and alloy composition with this technique. Therefore, we used HRTEM images to retrieve these structural parameters to verify our intended ternary structure. The observations were performed using an aberration (image) corrected Titan 80-300 TEM, which permits the SLs to be imaged at extremely high spatial resolution $(\approx 1 \AA)$, with negligible delocalization artifacts at interfaces. The inset of Fig. 1 is a typical HRTEM image of a compressively strained $(\varepsilon \sim+0.2 \%)$ ternary SL structure in the series, showing the first few SL periods adjacent to the substrate, wherein the amplitude contrast is optimized to delineate the individual layers in the structure. The average values for the individual layer thicknesses of InAs and GaInSb measured from this image were $44.1 \AA \pm 1.2 \AA$ and $24.1 \AA \pm 1.6 \AA$, respectively.

To examine the nature of transient interfaces, a detailed HRTEM study was performed, wherein the local strain distribution across individual layers in the SL was examined using techniques described in recent reports. ${ }^{21,22}$ The analysis was performed such that the strain component $\varepsilon_{\mathrm{xx}}$ was parallel to the interface (along [011]) and $\varepsilon_{\mathrm{yy}}$ along the growth direction ([100]). In Fig. 2, it is observed that the GaInSb layers (bright yellow regions) are in strong compressive strain (approximately 0.03 ), which is consistent with the high indium content in these layers, and that the InAs layers (bright green) are in tensile strain of about 0.01 , which is in agreement with theoretical calculations based on published values of elastic constants. The GaInSb-on-InAs and InAs-on-GaInSb interfaces are seen to exhibit strain inversion, so that the overall strain in these regions is negligible. Further analysis indicated that the net strain over several periods examined is about +0.02 , which is close to the measured value by XRD. 
(a)

(b)
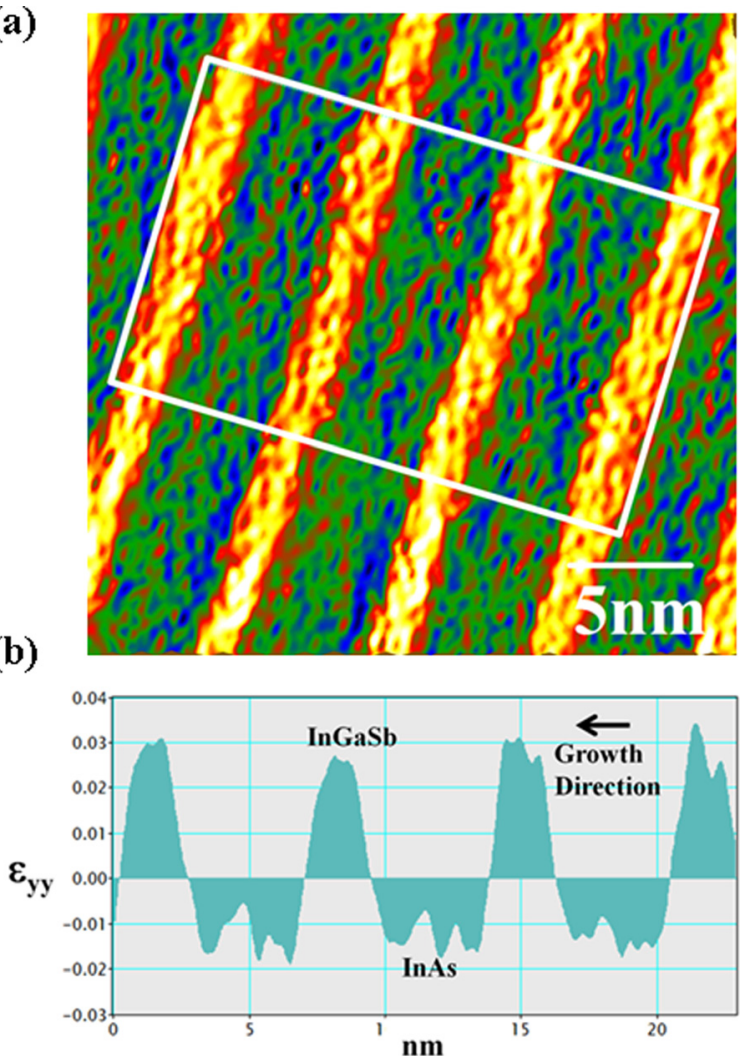

FIG. 2. (Color online) (a) Strain map of an $\mathrm{InAs} / \mathrm{Ga}_{0.75} \mathrm{In}_{0.25} \mathrm{Sb}$ superlattice and (b) the strain profile of the strain tensor $\varepsilon_{y y}$ along the growth direction averaged parallel to the interface within the white box in (a).

With the measured SL period of $68.0 \AA$, we obtained a band gap energy of $46 \mathrm{meV}$, or a corresponding onset wavelength of $27 \mu \mathrm{m}$ as demonstrated in the photoresponse (PR) spectrum in Fig. 3. A summary of the measured HRXRD SL period, net strains, and measured band gap energy determined from the onset of the PR spectra is plotted in Fig. 4. This

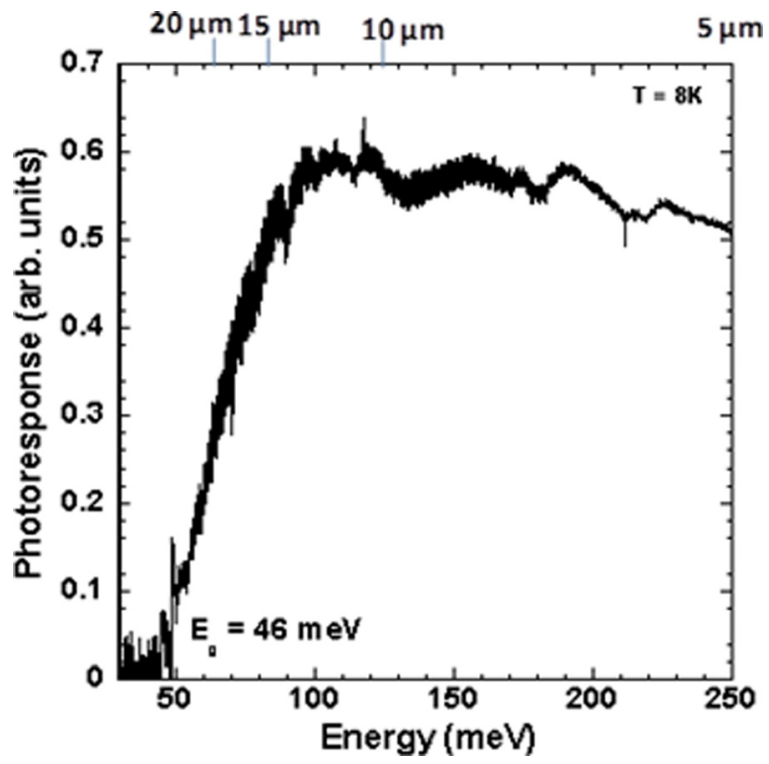

FIG. 3. (Color online) Photoresponse spectrum at $8 \mathrm{~K}$ for the $47.0 \AA$ InAs/21.5 $\AA \mathrm{Ga}_{0.75} \mathrm{In}_{0.25} \mathrm{Sb}$ superlattices.

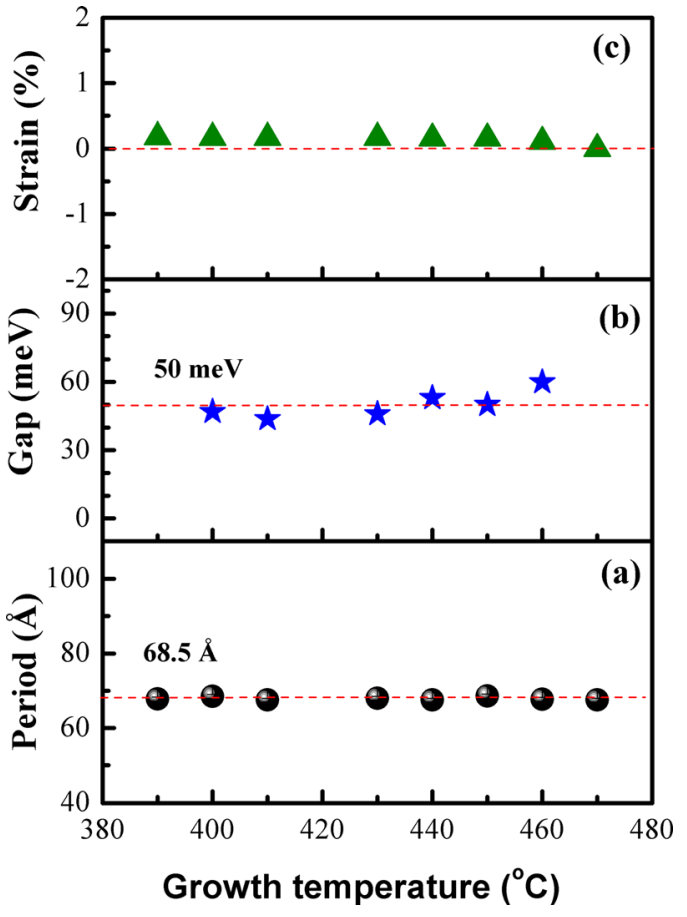

Fig. 4. (Color online) (a) Measured period (black circles), (b) measured band gap (blue stars), and (c) measured net strain (green triangle) as a function of the growth temperature.

figure demonstrates the repeatability of our MBE growth process over multiple sample depositions. The average period of grown samples in the series was $68.0 \AA \pm 0.5 \AA$, which is very close to the intended SL period of $68.5 \AA$. The measured SL structures with $68.0 \AA \pm 0.5 \AA$ periods produced a fairly consistent band gap around $50 \pm 5 \mathrm{meV}$.

\section{B. Morphological quality}

In order to evaluate the surface morphology of the grown samples, the surface roughness and the number of defects, as well as the size and shape, were monitored by AFM images scanned over $50 \mu \mathrm{m} \times 50 \mu \mathrm{m}$ areas. Figure 5 shows images of a few selected SL samples that were grown at $T_{\mathrm{g}}$ of 390 , 400,460 , and $470{ }^{\circ} \mathrm{C}$, respectively. Despite the large differences in $T_{\mathrm{g}}$, there were no significant changes in the average root-mean-square (RMS) roughness for the SL samples grown at $T_{\mathrm{g}}$ between 400 and $460{ }^{\circ} \mathrm{C}$. In this temperature range, the RMS values were around $3 \AA$ [see Fig. 6(a)]. However, the RMS roughness values for the two SL samples grown at the lowest $\left(390^{\circ} \mathrm{C}\right)$ and the highest temperature

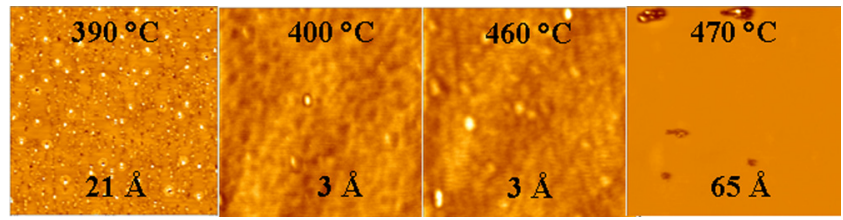

FIG. 5. (Color online) AFM images of $50 \mu \mathrm{m} \times 50 \mu \mathrm{m}$ area scans of $0.5 \mu \mathrm{m}$ thick 47.0 $\AA$ InAs/21.5 $\mathrm{Ga}_{0.75} \mathrm{In}_{0.25} \mathrm{Sb}$ superlattices grown at growth temperature $\left(\mathrm{T}_{\mathrm{g}}\right)$ of $390-470^{\circ} \mathrm{C}$ (from left to right). The value listed on the top (the bottom) of each image represents a $\mathrm{T}_{\mathrm{g}}$ (an average root-mean-square roughness). 


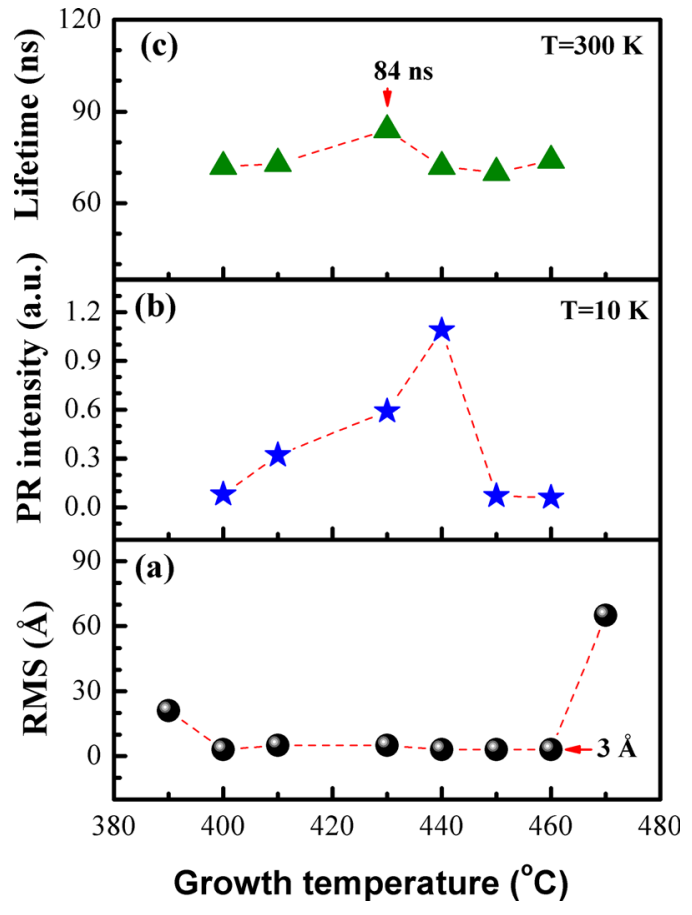

FIG. 6. (Color online) (a) Root mean square (RMS) of $50 \times 50 \mu \mathrm{m}^{2}$ scans in $\mathrm{AFM}$, (b) the photoresponse intensity measured at $100 \mathrm{meV}$ above the band gap, and (c) minority carrier lifetime measured at $300 \mathrm{~K}$ as a function of growth temperature, respectively, for $0.5 \mu \mathrm{m}$ thick $47.0 \AA$ InAs/21.5 $\mathrm{Ga}_{0.75} \mathrm{In}_{0.25} \mathrm{Sb}$ superlattice samples.

$\left(470^{\circ} \mathrm{C}\right)$ were significantly different from the others; their respective RMS values were 21 and $65 \AA$.

\section{Optical quality}

Photoresponse spectra were used to determine the SL band gap, to monitor the sharpness of the PR onset, and to determine the wavelength range covered. In addition, the intensity of the PR spectra was used as an indicator of the performance of the grown SL materials for infrared sensing. The spectra were collected with a Fourier transform infrared spectrometer at a temperature of $10 \mathrm{~K}$. Due to the relatively low resistivity of the samples, the photoconductivity was measured in the current-biased mode, with a current of $0.5 \mathrm{~mA}$ between two parallel strip contacts on the surface. The six PR spectra were collected from the sample set with $T_{\mathrm{g}}$ varying from 400 to $460{ }^{\circ} \mathrm{C}$, where the RMS roughness value was around $3 \AA$. Although the PR intensities are given in arbitrary units, the relative signal strengths can still be compared as the test conditions for all the samples were kept constant. We observe that the band gap energies of all SLs in this series were consistently around $50 \pm 5 \mathrm{meV}$, as shown in Fig. 4(b). The PR intensity measured at $100 \mathrm{meV}$ above the band gap is plotted in Fig. 6(b), where the intensity gradually increases as $T_{\mathrm{g}}$ increases from 400 to $440{ }^{\circ} \mathrm{C}$, reaching a maximum at $440^{\circ} \mathrm{C}$, and then drops by over an order of magnitude at higher growth temperatures. Thus, there is a relatively narrow growth window of $410-440{ }^{\circ} \mathrm{C}$ for the group $\mathrm{V}$ fluxes and cracking zone temperatures used. Outside this temperature window, the material quality deteriorates very rapidly.
The measured band gap energies from all of the grown SL ternary samples were approximately $30 \mathrm{meV}$ lower than the theoretically predicted value of $80 \mathrm{meV}$ by Grein et al. ${ }^{7}$ Correspondingly, the cutoff wavelength of $19 \mu \mathrm{m}$, determined at the point where the PR intensity has dropped by $50 \%$, was longer than the predicted $15 \mu \mathrm{m}$. Although the HRXRD and HRTEM measurements verify that the SL samples grown were very close to the intended design, there remains a discrepancy between theory and experiment that needs to be resolved. Overall, a good PR signal was measured despite the fact that the SL total thickness was only $0.5 \mu \mathrm{m}$, as demonstrated in Fig. 3. The strong fringing on the long wavelength portion of the spectrum is not noise but a result of multiple internal reflections through the substrate/ buffer/superlattice stack. The lightly doped n-type GaSb wafers do have significant infrared transparency. ${ }^{23}$ As shown in Fig. 3, the SL has a strong photoresponse over a wide wavelength range up to $\sim 15 \mu \mathrm{m}$.

\section{Carrier recombination quality}

Since the carrier lifetimes of the grown SL materials ultimately set the performance upper limits of the detectivity and operating temperatures, we used time-resolved differential reflectivity measurements to evaluate the recombination lifetimes of carriers at $300 \mathrm{~K}$ for the SL samples in the series. The pump was the output of an optical parametric amplifier (OPA), which was pumped by a regeneratively amplified Ti:Sapphire laser (CPA-2001, Clark-MXR, Inc.) with a repetition rate of $1 \mathrm{kHz}$. The wavelength of the OPA beam was tuned to $1.5 \mu \mathrm{m}$. The probe was the output of a continuouswave laser diode with a wavelength of $780 \mathrm{~nm}$. A silicon balanced detector with a bandwidth of $350 \mathrm{MHz}$ (PDB130A, Thorlabs, Inc.) was used to measure the difference in optical power between a reference arm of the probe beam, and the probe beam reflected from the surface of the sample at the pump spot. By measuring the RF output of the balanced detector with a fast oscilloscope, we were able to measure the change in reflectivity due to the pump with a time resolution of $\sim 1$ ns. Figure 7 shows the result of this measurement at $300 \mathrm{~K}$ for a typical ternary SL sample in the series. Here, we see multiple exponential decay components in the differential reflectivity, with the dominant contribution coming from an exponential with a decay time of $\sim 84 \mathrm{~ns}$. This should be interpreted as the lower limit for the actual recombination lifetime since the pump-probe signal is likely to be affected by carrier diffusion both in the lateral and vertical directions, ${ }^{24,25}$ which contributes to a faster decay of the signal. Minority carrier lifetimes measured at $300 \mathrm{~K}$ of the SL samples in this series are summarized in Fig. 6(c). We observe that the SL grown at $430{ }^{\circ} \mathrm{C}$ produced the longest lifetime of $84 \mathrm{~ns}$, but there were no significant changes in the overall lifetime for the ternary SL samples grown at $T_{\mathrm{g}}$ from 400 to $460^{\circ} \mathrm{C}$. In this temperature range, the average lifetime value was around $74 \mathrm{~ns}$, which is moderately long as compared to the most reported $300 \mathrm{~K}$ lifetime values from processed VLWIR detector materials. The summary of the results evaluated by AFM, photoconductivity, and time-resolved differential reflectivity measurements is plotted in Fig. 6 and listed in Table I. 


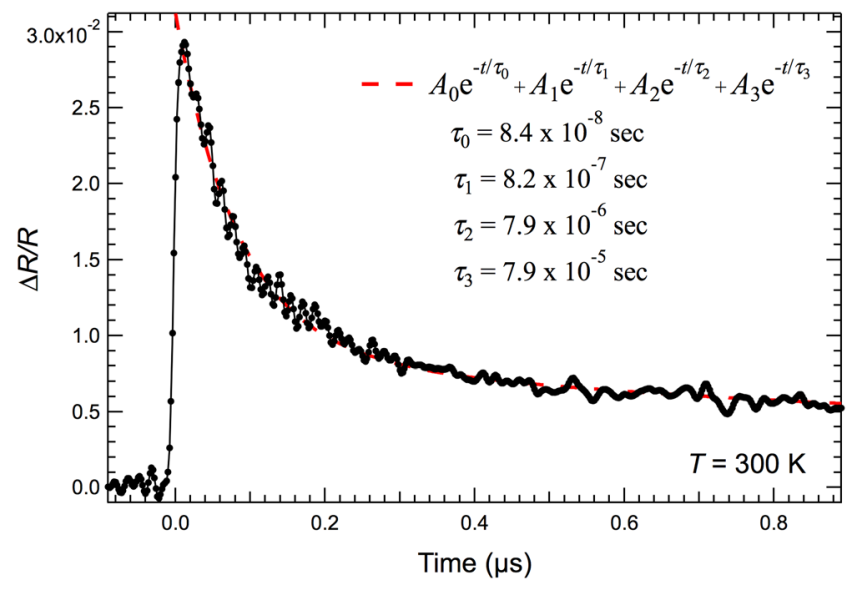

FIG. 7. (Color online) Time-resolved differential reflectivity data for a $47.0 \AA$ InAs/21.5 $\mathrm{Ga}_{0.75} \mathrm{In}_{0.25} \mathrm{Sb}$ superlattice sample grown at $430^{\circ} \mathrm{C}$ measured at $300 \mathrm{~K}$. The data is fit with multiple exponetials, $A_{0} e^{-t / \tau_{0}}$ $+A_{1} e^{-t / \tau_{1}}+A_{2} e^{-t / \tau_{2}}+A_{3} e^{-t / \tau_{3}}$, with the dominant exponential having a decay time of $\sim 84 \mathrm{~ns}$, where $A_{0}=1.2 \times 10^{-3} \pm 1 \times 10^{-4}, A_{1}=2.8 \times 10^{-4}$ $\pm 1 \times 10^{-5}, A_{2}=1.3 \times 10^{-4} \pm 1 \times 10^{-5}$, and $A_{3}=1.1 \times 10^{-4} \pm 1 \times 10^{-5}$.

\section{CONCLUSIONS}

In conclusion, through a combination of high-resolution $\mathrm{x}$-ray rocking curves, high-resolution transmission electron microscopy, atomic force microscopy, photoconductivity, and time-resolved differential reflectivity measurements, we investigated the impact of growth temperature on the quality of VLWIR InAs/GaInSb ternary SL materials. For the studies, a series of $47.0 \AA$ InAs/21.5 $\mathrm{Ga}_{0.75} \mathrm{In}_{0.25} \mathrm{Sb}$ SLs were grown by $\mathrm{MBE}$ at growth temperature $\left(T_{\mathrm{g}}\right)$ ranging from 390 to $470{ }^{\circ} \mathrm{C}$. The results showed that our MBE growth process produced a consistent band gap of $50 \pm 5 \mathrm{meV}$. However, the material quality of the grown samples, as assessed by various measurement techniques, was sensitive to $T_{\mathrm{g}}$. We observed a general trend of improving photoresponse intensity as $T_{\mathrm{g}}$ increases. The spectral photoresponse exhibited a maximum signal for the growth temperature of $440^{\circ} \mathrm{C}$, and then rapidly dropped at higher temperatures. The average surface roughness of SL samples that produce strong photoresponse spectra was around $3 \AA$, and a quantitative analysis

TABLE I. Summary of the measurement results for the sample set. The photoresponse and lifetime results are from measurements at $10 \mathrm{~K}$ and $300 \mathrm{~K}$, respectively. The cut-off wavelength $\lambda_{\mathrm{c}}$ is selected at the point where the intensity drops by $50 \%$. The PR intensity was measured at $100 \mathrm{meV}$ above the band gap. The average root-mean-square roughness was based on AFM images of $50 \times 50 \mu \mathrm{m}^{2}$ area scan.

\begin{tabular}{lcccccccc}
\hline \hline Sample & $\begin{array}{c}\mathrm{T}_{\mathrm{g}} \\
\left({ }^{\circ} \mathrm{C}\right)\end{array}$ & $\begin{array}{c}\mathrm{P} \\
(\AA)\end{array}$ & $\begin{array}{c}\varepsilon \\
(\%)\end{array}$ & $\begin{array}{c}\mathrm{E}_{\mathrm{g}} \\
(\mathrm{meV})\end{array}$ & $\begin{array}{c}\lambda_{\mathrm{c}} \\
(\mu \mathrm{m})\end{array}$ & $\begin{array}{c}\text { RMS } \\
(\AA)\end{array}$ & $\begin{array}{c}\text { PR int. } \\
(\text { a.u. })\end{array}$ & $\begin{array}{c}\text { Lifetime } \\
(\mathrm{ns})\end{array}$ \\
\hline 1 & 390 & 67.8 & +0.18 & $\mathrm{X}$ & $\mathrm{X}$ & 21 & $\mathrm{X}$ & $\mathrm{X}$ \\
2 & 400 & 68.5 & +0.17 & 47.0 & 19.0 & 3 & 0.08 & 72 \\
3 & 410 & 67.5 & +0.17 & 43.8 & 20.1 & 5 & 0.32 & 73 \\
4 & 430 & 68.0 & +0.17 & 46.0 & 19.0 & 5 & 0.59 & 84 \\
5 & 440 & 67.5 & +0.16 & 53.0 & 17.0 & 3 & 1.09 & 72 \\
6 & 450 & 68.6 & +0.16 & 50.0 & 16.2 & 3 & 0.07 & 70 \\
7 & 460 & 67.7 & +0.11 & 60.0 & 15.7 & 3 & 0.06 & 74 \\
8 & 470 & 67.5 & +0.00 & $\mathrm{X}$ & $\mathrm{X}$ & 65 & $\mathrm{X}$ & $\mathrm{X}$ \\
\hline \hline
\end{tabular}

of the strain distribution performed at the atomic scale by aberration corrected TEM provided valuable information about strain distribution at the interfaces. A strong compressive strain induced by GaInSb alloyed layers could compensate most tensile strain created by thick InAs layers, and the residual net strain was small around $+0.2 \%$. The SL grown at $430{ }^{\circ} \mathrm{C}$ produced the longest $300 \mathrm{~K}$ lifetime of $84 \mathrm{~ns}$.

\section{ACKNOWLEDGMENTS}

The work of H. J. Haugan was performed under Air Force contract number FA8650-11-D-5801. The authors thank S. Fenstermaker and G. Landis for a technical assistance with the MBE system and sample preparation for the measurements, respectively. The lifetime measurements reported here were conducted at Rice University with supported by by Air Force Research Laboratory under agreement No. FA8650-07-5601. G.T.N., N.E.O., and J.K. acknowledge support from the National Science Foundation through Grant No. EEC-0540832.

${ }^{1}$ C. Downs and T. E. Vandervelde, Sensors 13, 5054 (2013).

${ }^{2}$ L. Zheng, M. Z. Tidrow, A. Novello, H. Weichel, and S. Vohra, Proc. SPIE 6900, 69000F (2008).

${ }^{3}$ A. Rogalski, Infrared Phys. Technol. 50, 240 (2007).

${ }^{4}$ A. Rogalski, Prog. Quantum Electron. 27, 59 (2003).

${ }^{5}$ D. L. Smith and C. Mailhiot, J. Appl. Phys. 62, 2545 (1987).

${ }^{6} \mathrm{H}$. Kroemer, Physica E 20, 196 (2004).

${ }^{7}$ C. H. Grein, W. H. Lau, T. L. Harbert, and M. E. Flatté, Proc. SPIE $\mathbf{4 7 9 5}$ 39 (2002).

${ }^{8}$ Y. Wei, A. Gin, M. Razeghi, and G. J. Brown, Appl. Phys. Lett. 80, 3262 (2002).

${ }^{9}$ H. Mohseni, Y. Wei, and M. Razeghi, Proc. SPIE 4288, 191 (2001).

${ }^{10}$ Y. Wei, A. Gin, M. Razeghi, and G. J. Brown, Appl. Phys. Lett. 81, 3675 (2002).

${ }^{11}$ H. Mohseni, M. Razeghi, G. J. Brown, and Y. S. Park, Appl. Phys. Lett. 78, 2107 (2001).

${ }^{12}$ E. H. Aifer, E. M. Jackson, G. Boishin, L. J. Whitman, I. Vurgaftman, J. R. Meyer, J. C. Culbertson, and B. R. Bennett, Appl. Phys. Lett. 82, 4411 (2003).

${ }^{13}$ H. J. Haugan, S. Elhamri, F. Szmulowicz, B. Ullrich, G. J. Brown, and W. C. Mitchel, Appl. Phys. Lett. 92, 071102 (2008).

${ }^{14}$ H. J. Haugan, S. Elhamri, G. J. Brown, and W. C. Mitchel, J. Appl. Phys. 104, 073111 (2008).

${ }^{15}$ H. J. Haugan, S. Elhamri, W. C. Mitchel, B. Ullrich, G. J. Brown, L. Grazulis, and S. Houston, Proc. SPIE 7222, 72220 Y (2009).

${ }^{16}$ H. J. Haugan, G. J. Brown, and L. Grazulis, J. Vac. Sci. Technol. B 29, 03C101 (2011).

${ }^{17}$ E. M. Jackson, G. I. Boishin, E. H. Aifer, B. R. Bennett, and L. J. Whitman, J. Cryst. Growth 270, 301 (2004).

${ }^{18}$ B. Z. Nosho, B. B. Bennett, L. J. Whitman, and M. Goldenberg, J. Vac. Sci. Technol. B 19, 1626 (2001).

${ }^{19}$ R. H. Miles, D. H. Chow, Y.-H. Zhang, P. D. Brewer, and R. G. Wilson, Appl. Phys. Lett. 66, 1921 (1995).

${ }^{20}$ H. J. Haugan, G. J. Brown, S. Elhamri, W. C. Mitchel, K. Mahalingam, M. Kim, G. T. Noe, N. E. Ogden, and J. Kono, Appl. Phys. Lett. 101, 171105 (2012).

${ }^{21}$ K. Mahalingam, H. J. Haugan, G. J. Brown, K. G. Eyink, and Bin Jiang Proc. SPIE 8268, 826831 (2012).

${ }^{22}$ K. Mahalingam, H. J. Haugan, G. J. Brown, and K. G. Eyink, Ultramicroscopy 127, 70 (2013)

${ }^{23}$ L. P. Allen, P. Flint, G. Dallas, D. Bakken, K. Blanchat, G. J. Brown, S. R. Vangala, W. D. Goodhue, and K. Krishnaswami, Proc. SPIE 7298, 72983P (2009).

${ }^{24}$ H. J. Haugan, G. J. Brown, F. Szmulowicz, S. Elhamri, B. V. Olson, T. F. Boggess, and L. Grazulis, Proc. SPIE 8154, 81540J (2011).

${ }^{25}$ H. J. Haugan, G. J. Brown, S. Elhamri, S. Pacley, B. V. Olson, and T. F. Boggess, J. Appl. Phys. 111, 053113 (2012). 